\title{
Evaluación de la fiabilidad en instrumentos de valoración funcional en pacientes en hemodiálisis
}

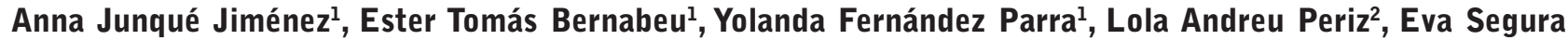 \\ Ortí \\ ${ }^{1}$ Unidad de Nefrología. Hospital de Terrassa. Consorci Sanitari de Terrassa. España \\ ${ }^{2}$ Departamento de Enfermería Fundamental y Medicoquirúrgica. Facultad de Medicina y Ciencias de la Salud. Uni- \\ versitat de Barcelona. España \\ ${ }^{3}$ Departamento de Fisioterapia.Universidad Cardenal Herrera-CEU, CEU Universities. Valencia. España
}

\section{Resumen}

Introducción: Cada vez es más frecuente promocionar programas de ejercicio físico en pacientes en hemodiálisis. Se puede valorar la condición física de estos pacientes y el resultado de dichos programas con diferentes pruebas funcionales.

Objetivo: Valorar la fiabilidad en términos de concordancia interobservador de las mediciones de capacidad funcional y fuerza muscular en pacientes en hemodiálisis.

Material y Método: 30 pacientes en hemodiálisis realizaron una batería de pruebas funcionales en dos fases: Short Physical Performance Battery, equilibrio estático monopodal, Timed Up and Go, Test Sit-to-stand-ToSit-5, Sit-to-stand-To-Sit-10, Sit-to-stand-To-Sit-60, fuerza de tríceps sural, 6 minutos marcha y dinamometría de la mano.

Resultados: El índice de correlación intraclase para la fiabilidad interobservador fue para el test Sit-to-standTo-Sit-5: 0,779; velocidad en 4 metros: 0,820; puntuación total Short Physical Performance Battery: 0,807;

\section{Correspondencia:}

Anna Junqué Jiménez

Nefrología Hospital de Terrassa. Consorci Sanitari de Terrassa Crta Torrebonica s/n 08227 Terrassa. Barcelona

E-mail: annajunque@yahoo.es
Sit-to-stand-To-Sit-10: 0,908; Sit-to-stand-To-Sit-60: 0,865; 6 minutos marcha: 0,897; Equilibrio monopodal: 0,925; Timed Up and Go: 0,918; Fuerza de tríceps sural derecho: 0,702; Fuerza de tríceps sural izquierdo: 0,995; dinamometría mano derecha con apoyo: 0,952; dinamometría mano izquierda con apoyo: 0,897; dinamometría mano derecha sin apoyo: 0,973; dinamometría mano izquierda con apoyo: 0,964.

Conclusiones: La fiabilidad interobservador en la mayoría de las pruebas es alta, por lo que se puede aceptar que la valoración del estado funcional del paciente y de los resultados de los programas destinados a promocionar el ejercicio lo lleven a cabo diferentes observadores experimentados, lo que facilitaría el seguimiento de los pacientes.

PALABRAS CLAVE: hemodiálisis; prueba ergométrica; capacidad de respuesta; fuerza muscular; fiabilidad.

\section{Evaluation of reliability in functional assessment tools in patients on hemodialysis}

\section{Abstract}

Introduction: It is increasingly common to promote physical exercise programs in hemodialysis patients. The physical condition of patients and the result of these programs with different functional tests can be assessed. 
Objective: To assess the reliability in terms of interobserver reliability of functional capacity and muscle strength measurements in hemodialysis patients.

Material and Method: 30 patients on hemodialysis performed several functional tests in two phases: Short Physical Performance Battery, static monopodal equilibrium, Timed Up and Go, Test Sit-to-stand-ToSit-5, Sit-to-stand-To-Sit-10, Sit-to-stand-To-Sit-60, strength of triceps sural, 6 minutes walking and dynamometry of the hand.

Results: The intraclass correlation coefficient for interobserver reliability was for the Sit-to-stand-ToSit-5 test: 0.779; speed in 4 meters: 0.820; Total score Short Physical Performance Battery: 0.807; Sit-to-stand-To-Sit-10: $\quad 0.908$; Sit-to-stand-ToSit-60: $0.865 ; 6$ minutes march: 0.897; Monopodal equilibrium: 0.925; Timed Up and Go: 0.918; Right triceps sural force: 0.702 ; Left triceps sural force: 0.995; Right hand dynamometry with support: 0.952 ; Left hand dynamometry with support: 0.897; right hand dynamometry without support: 0.973 ; left hand dynamometry with support: 0.964 .

Conclusions: The interobserver reliability, in most tests is high, so it can be accepted that the assessment of the patient's functional status and the results of the programs designed to promote the exercise is carried out by different experienced observers, which would facilitate the follow-up of patients.

KEYWORDS: hemodialysis; ergometric test; capacity to respond; muscular strength; reliability.

\section{Introducción}

Un aspecto de gran importancia en los pacientes con IRCT (Insuficiencia renal crónica terminal), es la disminución de su condición física a medida que van pasando los años ${ }^{1-5}$.

La edad, la malnutrición, la anemia, la inflamación crónica, las alteraciones del metabolismo óseo mineral, así como una elevada comorbilidad asociada y las propias alteraciones del metabolismo de la urea, podrían ser algunos de los factores que contribuyen a un empeoramiento progresivo de su estado general y que nos llevará con el paso de los días, a un estado de debilidad muscular y con los años a un posible paso de independencia a dependencia para las actividades de la vida cotidiana ${ }^{6}$

Desde principios de la década de 1980, países como Estados Unidos comenzaron a implementar programas de ejercicio físico durante las sesiones de hemodiálisis (HD) con el objetivo de mejorar la calidad de vida relacionada con la salud. Cada vez es más frecuente la literatura donde nos muestra que la realización de un programa de ejercicio físico mejora la fuerza muscular, la capacidad funcional y la calidad de vida relacionada con la salud (CVRS) de estos pacientes, incluso en la población de edad más avanzada ${ }^{7-10}$.

Los efectos de dichos programas se han medido con una gran variedad de pruebas. La utilización de estas pruebas es muy importante para poder generalizar el uso de herramientas válidas de evaluación, y para obtener información fiable tanto del deterioro producido en la capacidad funcional por la enfermedad y la edad, así como del efecto beneficioso que puedan presentar los programas de ejercicio físico para mejorar la funcionalidad ${ }^{5,11}$.

En la actualidad, no existe en la literatura el análisis de la fiabilidad en términos de concordancia interobservador de estas pruebas en pacientes en HD. Pensamos que resultaría interesante evaluar si la toma de variables de capacidad funcional y fuerza muscular pueden verse afectadas dependiendo de los diferentes profesionales que realicen la prueba a una misma persona utilizando el mismo protocolo. Por lo tanto, nos planteamos el objetivo de valorar la fiabilidad en términos de concordancia interobservador de las mediciones de capacidad funcional y fuerza muscular en pacientes en hemodiálisis utilizando el mismo protocolo.

\section{Material y Método}

Entre los meses de enero y febrero del 2018, se realizó un estudio observacional de 4 semanas de duración, para valorar la fiabilidad en términos de concordancia interobservador de las mediciones de capacidad funcional y fuerza muscular en pacientes en HD con la utilización del mismo protocolo.

El servicio de Nefrología del Consorci Sanitari de Terrassa consta de una población de 65 pacientes en HD. Se incluyeron todos los pacientes de la unidad y se se- 
leccionaron para el estudio aquellos que cumplían los criterios de inclusión.

Como criterios de inclusión se establecieron: Estar al menos 3 meses en tratamiento de HD, encontrarse en situación de estabilidad clínica y hemodinámica y otorgar el consentimiento informado por escrito. Por otra parte, los criterios de exclusión establecidos fueron: Infarto de miocardio en las 6 semanas previas, angina inestable al ejercicio o en reposo, amputación de miembros inferiores sin prótesis, enfermedad vascular cerebral (ictus, isquemias transitorias) en las 6 semanas previas, alteraciones musculoesqueléticas o respiratorias que empeoren con el ejercicio, imposibilidad física manifiesta de realizar las pruebas funcionales y no otorgar el consentimiento informado.

Variables analizadas:

- Datos de la Historia Clínica: Edad, sexo, etiología de la IRCT. Se recopilaron, además, los principales datos bioquímicos de la analítica trimestral realizada de forma habitual en los controles de los pacientes en HD.

- Pruebas físicas funcionales: Se valoró a los sujetos con dos baterías de pruebas funcionales encaminadas a evaluar la condición física y la fuerza de los pacientes.

El estudio consistió en repetir las mismas pruebas de condición física y fuerza muscular en dos fases. Cada fase se realizó por profesionales diferentes y expertos en la toma de estas pruebas. La realización se hizo en el momento previo a que el paciente entre a su sesión habitual de HD y según un protocolo establecido.

\section{Definición de las pruebas:}

Las pruebas se realizaron durante la semana debido a problemas en las dinámicas de los servicios de HD, la falta de tiempo y recursos humanos (Tabla 1 ).

\section{a) Primera batería de pruebas funcionales:}

1.- La prueba "Short Physical Performance Battery" (SPPB) se registró en todos los sujetos el primer día antes de la sesión de $\mathrm{HD}^{7,12}$.

- Consta de tres pruebas de equilibrio con los pies juntos, en posición de semi-tándem y en tándem. Se valoró si el sujeto puede mantener cada una de estas posiciones hasta un máximo de 10 segundos.
La valoración del equilibrio va de 0 a 4 puntos.

- Incluye también una prueba de velocidad de marcha en 4 metros a velocidad habitual. Se realizaron dos intentos cronometrados y se registró el mejor de ellos, de forma que según los segundos empleados se asigna una puntuación de 1 a 4 .

- Se valoró también la prueba STS-5, que consiste en medir el tiempo empleado en levantarse y sentarse de la silla 5 veces, el tiempo se detiene cuando el paciente alcanza la posición de pie en la repetición 5 . Según el tiempo empleado se asigna una puntuación de 0 a 4 puntos.

Por lo tanto, la puntuación del SPPB va de 1 a 12 puntos.

2.- El equilibrio estático monopodal, se valoró previamente a la primera sesión de hemodiálisis; pidiendo a los sujetos que levanten una pierna flexionándola de forma que se pongan a la pata coja (se le dice que la pierna con la que se sienta más seguro). Se les pidió que aguantaran el equilibrio el mayor tiempo posible. El investigador apuntó el tiempo que el paciente aguantó en posición de apoyo monopodal. El sujeto podía mover los brazos y flexionar la rodilla si lo necesitaba para mantener el equilibrio. El tiempo finalizó cuando el sujeto usaba sus brazos para apoyarse, utilizó el pie elevado para apoyarse en el suelo, cuando el pie apoyado se movía para mantener el equilibrio o cuando el tiempo llegaba a los 45 segundos. Se repitió el procedimiento 3 veces y se registró el mejor tiempo ${ }^{13}$.

3.- La prueba "Timed Up and Go" (TUG), se realizó previamente a la primera sesión de HD. Desde una silla de altura estándar sin apoyabrazos, el paciente se levantó, anduvo 3 metros y volvió a la posición inicial. Se registró el tiempo y el grado de dificultad según la escala de esfuerzo percibido (EEP) ${ }^{14}$.

\section{b) Segunda batería de pruebas funcionales:}

1.- Las pruebas del "Sit to stand to sit 10 " (STS10) y "Sit to Stand to sit 60" (STS-60) se realizaron inmediatamente antes del segundo día de la sesión semanal de HD. La primera consistió en medir los segundos que necesitaba el paciente para, desde una posición 
Tabla 1. Esquema de trabajo.

\begin{tabular}{|c|c|c|c|c|}
\hline Día & Test & Definición & Puntuación / Registro & Total \\
\hline \multirow[t]{7}{*}{ Día 1} & \multirow{5}{*}{$\begin{array}{c}\text { Short Physical Perdormance } \\
\text { Battery (SPPB) } \\
\text { Puntuación total } 12 \text { puntos }\end{array}$} & Equilibrio pies juntos & $\begin{array}{l}0 \text { puntos }=0 \text { a } 9 \text { segundos } \\
1 \text { punto }=10 \text { segundos }\end{array}$ & 4 \\
\hline & & Equilibrio semi tandem & $\begin{array}{l}0 \text { puntos }=0 \text { a } 9 \text { segundos } \\
1 \text { punto }=10 \text { segundos }\end{array}$ & \\
\hline & & Equilibrio tandem & $\begin{array}{l}0 \text { puntos }=0 \text { a } 2 \text { segundos } \\
1 \text { punto }=3 \text { a } 9 \text { segundos } \\
2 \text { puntos }=10 \text { segundos }\end{array}$ & \\
\hline & & $\begin{array}{l}\text { Velocidad de la marcha } 4 \mathrm{~m} . \\
\text { Caminar } 4 \text { metros }\end{array}$ & $\begin{array}{l}1 \text { punto }=\geq 8,70 \text { segundos } \\
2 \text { puntos }=6,21-8,70 \text { segundos } \\
3 \text { puntos }=4,82-6,20 \text { segundos } \\
4 \text { puntos }=\leq 4,82 \text { segundos }\end{array}$ & 4 \\
\hline & & $\begin{array}{l}\text { STS5. Levantarse y sentarse en la } \\
\text { silla } 5 \text { veces, el tiempo se detiene } \\
\text { cuando el paciente alcanza la posi- } \\
\text { ción de pie en la repetición } 5\end{array}$ & $\begin{array}{l}0 \text { puntos }=\geq 60 \text { segundos } \\
1 \text { punto }=\geq 16,70 \text { segundos } \\
2 \text { puntos }=13,70-16,69 \text { segundos } \\
3 \text { puntos }=11,20-13,69 \text { segundos } \\
4 \text { puntos }=\leq 11,19 \text { segundos }\end{array}$ & 4 \\
\hline & El equilibrio estático monopodal & $\begin{array}{l}\text { Levantar una pierna flexionándola } \\
\text { de forma que se ponga a la pata coja }\end{array}$ & $\begin{array}{l}\text { Registro de la puntuación en segun- } \\
\text { dos. Máximo } 45 \text { segundos }\end{array}$ & \\
\hline & "Timed Up and Go" (TUG) & $\begin{array}{l}\text { Desde una silla sin apoyabrazos, el } \\
\text { paciente se levanta, anda } 3 \text { metros } \\
\text { y vuelve a la posición inicia }\end{array}$ & Registro del tiempo en segundos & \\
\hline \multirow[t]{5}{*}{ Día2 } & STS10 & $\begin{array}{l}\text { Levantarse y volver a sentarse } 10 \\
\text { veces consecutivas }\end{array}$ & Registro del tiempo en segundos & \\
\hline & STS60 & $\begin{array}{l}\text { El máximo número de repeticiones } \\
\text { de levantarse y volver a sentar- } \\
\text { se a la silla en un tiempo de } 60 \\
\text { segundos }\end{array}$ & Registro del tiempo en segundos & \\
\hline & Tricep sural & $\begin{array}{l}\text { Elevar el talón todo lo que se pueda } \\
\text { al ritmo del metrónomo }\end{array}$ & $\begin{array}{l}\text { Registro de las repeticiones. Máxi- } \\
\text { mo } 25 \text { repeticiones }\end{array}$ & \\
\hline & HG sin apoyo & $\begin{array}{l}\text { Apretar dinamómetro. Sentados } \\
\text { en una silla, con los pies apoyados } \\
\text { en el suelo y las rodillas flexiona- } \\
\text { das } 90^{\circ} \text {. El hombro del brazo que } \\
\text { está registrando permaneció en } \\
\text { rotación neutra, codo en flexión de } \\
90^{\circ} \text { sin apoyo en la mesa, muñeca } \\
\text { y antebrazo semipronados }\left(0-30^{\circ}\right) \\
\text { y una desviación cubital entre } 0 \text { y } \\
15^{\circ} \text {. Sin apoyo }\end{array}$ & Registro en kg & \\
\hline & HG con apoyo & $\begin{array}{l}\text { Apretar dinamómetro. Sentados } \\
\text { en una silla, con los pies apoyados } \\
\text { en el suelo y las rodillas flexiona- } \\
\text { das } 90^{\circ} \text {. El hombro del brazo que } \\
\text { está registrando permaneció en } \\
\text { rotación neutra, codo en flexión de } \\
90^{\circ} \text { apoyado en la mesa, muñeca } \\
\text { y antebrazo semipronados }\left(0-30^{\circ}\right) \\
\text { y una desviación cubital entre } 0 \text { y } \\
15^{\circ} \text {. Apoyado en una mesa }\end{array}$ & Registro en kg & \\
\hline Día3 & 6MWT & $\begin{array}{l}\text { Durante } 6 \text { minutos recorrer la } \\
\text { máxima distancia a un ritmo activo }\end{array}$ & Registro en metros & \\
\hline
\end{tabular}


de sentado, levantarse y volver a sentarse 10 veces consecutivas. El test se realizó en una silla sin apoyabrazos de unos $44,5 \mathrm{~cm}$ de altura, $38 \mathrm{~cm}$ de profundidad apoyada en la pared para minimizar el riesgo de caída durante la prueba. Se le permitió al paciente un intento de prueba, indicándole que los brazos debían permanecer cruzados frente al pecho. Tras realizar las 10 repeticiones se registró el tiempo empleado y se registró la escala de esfuerzo percibido (EEP). A continuación, el paciente descansaba mientras se le explicaba la prueba STS-60. Se explicaba al paciente que el test consistía en realizar el máximo número de repeticiones de levantarse y volver a sentarse a la silla en un tiempo de 60 segundos. Tras ese tiempo se anotaban las repeticiones y se registraba el grado de dificultad de la prueba según la EEP12.

2.- La fuerza de tríceps sural con la prueba de elevación de talón monopodal. Se realizó también inmediatamente antes de la segunda sesión de HD semanal. La prueba se utilizaba para valorar la fuerza funcional del tríceps sural de cada pierna y era realizada sin calzado. El ritmo de elevación del talón se marcaba mediante un metrónomo. Antes de iniciar la prueba se pedía al paciente que mantuviera el equilibrio sobre una sola pierna, con un apoyo ligero de las puntas de los dedos en la pared, con los brazos separados del tronco y evitando trasladar el peso a través de los brazos a la pared, mientras el pie contralateral se situaba ligeramente elevado del suelo. Se permitía un intento de elevación con el pie izquierdo, tras el cual se procedía a valorar la elevación del talón derecho. Se les pedía a los sujetos que elevaran el talón todo lo que podían al ritmo del metrónomo hasta que no podían elevar el talón por fatiga muscular. Si el sujeto compensaba con los brazos contra la pared o flexionaba la rodilla terminaba la prueba. También finalizaba si se alcanzaban 25 repeticiones, pues se has establecido que estas son las repeticiones que de media se encuentran en la población sana. Se registró al finalizar la prueba el número de repeticiones por cada pierna, así como el grado de dificultad según EEP11.

3.- La prueba de "Six minutes walking test" (6MWT), se realizó previamente a la terce- ra sesión semanal de $H D$, en el pasillo de la unidad. El paciente debió de acudir con zapatillas cómodas. En el momento previo a la realización de la prueba y, tras permanecer sentado 5 minutos, se registró la frecuencia cardiaca basal y la tensión arterial mediante un esfigmomanómetro digital en el brazo sin fístula. A continuación, se le indicó al paciente que, durante 6 minutos recorriera la máxima distancia a un ritmo activo. La orden dada al paciente era la siguiente: 'camine la mayor distancia posible en un tiempo de 6 minutos'. Se permitió realizar el test con ayudas para la deambulación si el paciente las utilizaba en su vida diaria o con la asistencia de otra persona, o parar y reiniciar la marcha en caso de necesitar un descanso durante la prueba. Se estandarizó la información sobre tiempo transcurrido, la forma y los momentos en que se animó al paciente de acuerdo con la literatura. Inmediatamente después de la finalización del test, se tomó el pulso y la tensión arterial. Se registró la distancia recorrida en metros y se pidió al paciente que describiera EEP15.

Evaluación de fuerza muscular: Se realizó la prueba de dinamometría de mano (Dinamómetro JAMAR), ya que hay estudios en pacientes en diálisis que correlacionan la fuerza de prensión de la mano con su estado de salud.

Esta valoración se realizó antes de la segunda sesión de HD semanal. Los sujetos se situaron sentados en una siIla, con los pies apoyados en el suelo y las rodillas flexionadas $90^{\circ}$. El hombro del brazo que está registrando permaneció en rotación neutra, codo en flexión de $90^{\circ}$ apoyado o sin apoyo en la mesa, muñeca y antebrazo semipronados $\left(0-30^{\circ}\right)$ y una desviación cubital entre 0 y $15^{\circ}$. Se realizaron 3 repeticiones consecutivas, con un descanso de 15 segundos entre ellas, en cada uno de los miembros superiores, comenzando por el brazo dominante. Se dio ánimo verbal en las repeticiones y se registró la de valor máximo ${ }^{11}$.

Este estudio fue aprobado por el Comité Ético de nuestra institución y realizado de acuerdo con las normas de la Declaración de Helsinki.

El estudio se realizó mediante la elaboración de una base de datos restringida en formato Excel 2010 para Windows ${ }^{\circledR}$, con estricto cumplimiento de las normas vigentes en relación con la confidencialidad de datos 
(LOPD; Ley Orgánica 15/1999 de 13 de diciembre de Protección de Datos de Carácter Personal).

El contenido de la base de datos fue transferido al paquete estadístico SPSS (IBM SPSS Statistics 20). Se procedió al análisis estadístico realizando un análisis descriptivo de las variables de estudio presentando los resultados de las variables cualitativas como frecuencias absolutas y relativas y las variables cuantitativas como media y desviación estándar. Para analizar la concordancia interobservador se utilizó el cálculo de coeficiente de correlación interclase (ICC), con intervalo de confianza del $95 \%$.

\section{Resultados}

Se analizó a 65 pacientes en programa de HD en nuestra unidad. De ellos, 30 pacientes superaron los criterios establecidos y fueron incluidos $(66,7 \%$ hombres), con una edad media de $66,37 \pm 16,32$ años y un tiempo medio de permanencia en HD de $34,4 \pm 51,4$ meses. EI Índice de Charlson Medio fue de $8,5 \pm 2,5$. Los datos demográficos y analíticos se muestran en la Tabla 2 y Tabla 3 respectivamente.

Tabla 2. Datos demográficos, comorbilidad y principal etiología de la enfermedad renal.

\begin{tabular}{lc}
\hline Edad (años) & $66,37 \pm 16,3$ \\
Tiempo en HD (meses) & $34,4 \pm 51,4$ \\
Sexo (\% hombres) & 66,7 \\
Índice de Charlson & $8,5 \pm 2,5$ \\
Glomerulonefritis (\%) & 13,3 \\
Vascular (\%) & 13,3 \\
Diabetes Mellitus (\%) & 23,3 \\
\hline $\mathrm{n}=30$ & \\
\hline
\end{tabular}

En la Tabla 4 podemos ver los valores de la fuerza de concordancia según los valores del ICC, para poder valorar nuestros resultados. En nuestros resultados hemos obtenido como valor más bajo la prueba del STS-5 con una fuerza de concordancia del 0,779 y la fuerza del tríceps sural derecho con 0,702 . Por lo contrario, hemos obtenido como mayor puntuación los resultados de la fuerza muscular del tríceps sural izquierdo con un valor de 0,995 y de la fuerza de agarre en el brazo derecho sin apoyo con un valor de 0,973. La Tabla 5 muestra los resultados del ICC para la fiabilidad inter observador.
Tabla 3. Parámetros bioquímicos y nutricionales.

\begin{tabular}{|c|c|}
\hline \multicolumn{2}{|l|}{ Datos bioquímicos } \\
\hline Glucosa (mg/dl) & $133,65 \pm 55,75$ \\
\hline Creatinina (mg/dl) & $8,2 \pm 3,1$ \\
\hline $\mathrm{k}(\mathrm{mEq} / \mathrm{l})$ & $5,5 \pm 0,6$ \\
\hline $\mathrm{Ca}(\mathrm{mg} / \mathrm{dl})$ & $9,2 \pm 0,7$ \\
\hline$P(m g / d l)$ & $4,7 \pm 1,4$ \\
\hline i-PTH (pg/ml) & $499,5 \pm 664,2$ \\
\hline 25-0H VitD (ng/ml) & $23,9 \pm 9,6$ \\
\hline \multicolumn{2}{|l|}{ Parámetros nutricionales } \\
\hline Albúmina ( $\mathrm{g} / \mathrm{dl})$ & $3,8 \pm 0,4$ \\
\hline Colesterol total (mg/dl) & $153,2 \pm 47,2$ \\
\hline Colesterol HDL (mg/dl) & $42,2 \pm 14,9$ \\
\hline Colesterol LDL (mg/dl) & $78,4 \pm 29,3$ \\
\hline Triglicéridos (mg/dl) & $160,3 \pm 135,1$ \\
\hline Valoración nutricional objetiva & $26,53 \pm 4,1$ \\
\hline \multicolumn{2}{|l|}{ Datos hemograma } \\
\hline Hemoglobina (g/dl) & $11,5 \pm 1,3$ \\
\hline Ferritina (ng/ml) & $378,6 \pm 198,7$ \\
\hline \multicolumn{2}{|l|}{ Adecuación de diálisis } \\
\hline Dosis diálisis (Kt/v) & $1,8 \pm 0,6$ \\
\hline
\end{tabular}

Hemodiálisis $n=30$, k: potasio, Ca: Calcio, P: fósforo, i-PTH: hormona paratiroidea intacta, VitD: vitamina D, HDL: lipoproteinas de alta intesidad, LDL: lipoproteínas de baja intesidad, kt/v: método $2^{\mathrm{a}}$ generación Daurgirdas.

Tabla 4. Valoración de la concordancia según los valores del Coeficiente de Correlación Interclase.

\begin{tabular}{lc}
\hline Valor del ICC & Fuerza de la concordancia \\
\hline$\geq 0,90$ & Muy buena \\
\hline $0,71-0,90$ & Buena \\
\hline $0,51-0,70$ & Moderada \\
\hline $0,31-0,50$ & Mediocre \\
\hline$\leq 0,30$ & Mala o nula \\
\hline \hline
\end{tabular}

ICC: coeficiente correlación interclase.

\section{Discusión}

Es conocido que los pacientes renales muestran una disminución de la fuerza muscular y su capacidad funcional ${ }^{1-3}$. Aunque se ha evolucionado mucho en las técnicas dialíticas, aún no se ha conseguido atenuar éstos mencionados efectos adversos sobre el paciente renal. Por eso ya desde la década de los ochenta se empezaron a implementar programas de ejercicio físico de cara a evitar el deterioro funcional provocado por la IRCT ${ }^{10}$. 
Tabla 5. Coeficiente de correlación interclase para la fiabilidad interobservador.

\begin{tabular}{lcc}
\hline Variable & ICC & $95 \%$ \\
Puntuación total SPPB & 0,807 & $0,633-0,903$ \\
STS-5 & 0,779 & $0,586-0,888$ \\
Velocidad marcha 4 metros & 0,820 & $0,656-0,910$ \\
STS-10 & 0,908 & $0,817-0,955$ \\
STS-60 & 0,865 & $0,736-0,933$ \\
6MWT & 0,897 & $0,796-0,950$ \\
Equilibrio monopodal & 0,925 & $0,849-0,964$ \\
TUG & 0,918 & $0,834-0,960$ \\
Fuerza trícep sural derecho & 0,702 & $0,462-0,846$ \\
Fuerza trícep sural izquierdo & 0,995 & $0,990-0,998$ \\
HG derecho con apoyo & 0,952 & $0,902-0,977$ \\
HG izquierdo con apoyo & 0,897 & $0,796-0,950$ \\
HG derecho sin apoyo & 0,973 & $0,944-0,987$ \\
HG izquierdo sin apoyo & 0,964 & $0,925-0,983$ \\
\hline
\end{tabular}

$\mathrm{n}=30$. SPPB: Short Physical Performance Battery, STS-5: Test sit to stand to sit 5, STS-10 y 60: test to stant to sit 10 y 60, 6MWT: test 6 minutos marcha, TUG: time up and go, HG: Hand grip.

En el ámbito nacional, los programas de ejercicio físico centrados en el paciente renal se van implementando de forma paulatina, si bien en estos últimos años se han visto equipos de investigación muy centrados en esta práctica ${ }^{7-9,16}$.También es conocido el equipo del Consorci Sanitari de Terrassa, que publicó diversos estudios con programas de ejercicios intradiálisis de fuerza y resistencia así como de electroestimulación neuromuscular donde se muestra la efectividad de estos programas incluso con personas de edad avanzada ${ }^{8,9,18}$. Consideramos también importante mencionar la creación en mayo del 2018 del grupo GEMEFER (grupo español multidisciplinar de ejercicio físico en el enfermo renal) en la Sociedad Española de Nefrología (SEN) donde uno de sus objetivos es el desarrollar entre los profesionales sanitarios y pacientes renales iniciativas destinadas a la implantación de programas ejercicio físico para enfermos renales en cualquier estadio de la enfermedad.

Al ser una práctica novedosa y poco implementada, un obstáculo sobreañadido es la presencia de poblaciones y muestras muy limitadas, que dificultan la evaluación de la efectividad de dichos programas, así como intentar protocolizar de forma adecuada las evaluaciones funcionales para evidenciar su efectividad en la fuerza muscular y ca- pacidad funcional de los pacientes con IRCT. Revisando la literatura, existen algunas publicaciones científicas en la misma línea de nuestro trabajo donde se observa la fiabilidad absoluta y relativa de las pruebas funcionales en pacientes en $\mathrm{HD}^{11,16}$. De este modo con estas publicaciones se pueden valorar las mejores pruebas para poder evaluar la efectividad de los programas y la elección de las pruebas más fiables. Sin embargo, hay escasez de datos que muestren la concordancia interobservador en estas pruebas y estos pacientes.

Por este motivo, consideramos importante realizar nuestro estudio ya que se centra en la fiabilidad de las mediciones de capacidad funcional y fuerza muscular de los pacientes en HD valorando la concordancia interobservador, refiriéndose, a la consistencia entre dos observadores distintos cuando evalúan una misma medida en un mismo individuo $0^{19,20}$.

En las áreas de nefrología es muy difícil que un mismo profesional pueda medir siempre al mismo paciente, por la logística de los servicios y la falta de recursos humanos, de aquí la importancia de este proyecto y su divulgación.

Los principales hallazgos de este estudio fueron que la valoración de la concordancia según los valores de Coeficiente de Correlación Interclasse de las pruebas fue buena y muy buena. Todas las medidas tienen valores por encima de 0,71 .

Los factores que pueden explicar el alto resultado del ICC en todas las pruebas es que se ha realizado por dos profesionales expertos en estas pruebas y habituados, siguiendo un protocolo muy estricto de actuación y que también se ha seguido un estándar en las pruebas (mismo día y hora de la semana). Por lo tanto, podemos afirmar que realizando un mismo protocolo y con los profesionales formados se pueden obtener resultados muy fiables.

Se encontró en la literatura un artículo publicado en abril de este mismo año 2018, donde se evalúa la ICC en pacientes con IRC, pero no sometidos a HD. Sus resultados en ICC también son buenos, pero a diferencia de los nuestros, ellos en el STS-60 tienen una fuerza de concordancia muy buena $(0,927)$ y nosotros buena $(0,865)$ y en cambio en el STS-5 nosotros hemos obtenido una fuerza de concordancia buena $(0,77)$ y ellos solo moderada $(0,676)^{21}$.

La principal limitación de este estudio es el tamaño muestral, ya que, aunque teniendo una población de 65 pacientes muchos quedaron excluidos. 
Por otra parte, se realizaron las pruebas funcionales en una semana, estableciendo un protocolo. Es importante que, si se realizan posteriores estudios, seguir el mismo protocolo al realizar las pruebas y el mismo orden y día. Se realizó de esta manera debido a las organizaciones de las unidades de hemodiálisis, por la logística de los pacientes y por necesidades de los servicios (horarios y falta de personal).

Afirmar que el grupo investigador ha realizado toda una serie de pruebas funcionales, sin embargo, en los controles rutinarios de los pacientes no sería necesario realizar todas las pruebas, sino aquellas que cada servicio creyera necesario, por lo tanto, comentar que el 6MWT, el TUG, HG y SPPB son las pruebas funcionales más utilizadas en la población de estudio por diferentes autores ${ }^{9,11,18}$. Por otra parte, tríceps sural y equilibrio mono podal no son tan utilizados.

En vista de los resultados obtenidos podemos concluir, la fiabilidad interobservador, en la mayoría de las pruebas es alta por lo que se puede aceptar que la valoración del estado funcional del paciente y de los resultados de los programas destinados a promocionar el ejercicio lo lleven a cabo diferentes observadores experimentados lo que facilitaría el seguimiento de los pacientes.

Nuestros resultados animan a realizar futuros estudios multicéntricos para poder evaluar la capacidad funcional y la fuerza muscular de los pacientes y poder evaluar los programas de ejercicio físico en pacientes renales con muestras más grandes.

\section{Conflicto de intereses}

Los autores no declaran tener ningún conflicto de intereses.

\section{Agradecimientos}

A todos los pacientes por su valiosa colaboración en la presente investigación.

Este trabajo de investigación se ha realizado en el marco de programa de Doctorado en Enfermería y Salud de la Universidad de Barcelona (UB).
Recibido: 15 agosto 2018

Revisado: 18 agosto 2018

Modificado: 20 agosto 2018

Aceptado: 21 agosto 2018

\section{Bibliografía}

1. Ortega Pérez de Villar L, Antolí García S, Lidón Pérez MJ, Amer Cuenca JJ, Martínez Gramage J, Segura Ortí E. Cuantificación del deterioro funcional durante seis meses en pacientes renales en estadio terminal. Enferm Nefrol. 2015;18(4):265-71.

2. Hernández Sánchez $S$, García López D, Santos Lozano A, González-Calvo G, Brazález Tejerina M, Garatachea Vallejo N. Valoración física, condición física y calidad de vida en pacientes con diferentes tratamientos renales sustitutivos. Enferm Nefrol. 2015;18(2):81-8.

3. Barbero Narbona E, Tejeda Araez E, Herrera Morales C, Montserrat García S, Gascó Coscojuela N, Junyent Iglesias E. Estudio comparativo del estado físico, mental y percepción de calidad de vida relacionada con la salud de los pacientes en diálisis. Enferm Nefrol. 2016;19(1):29-35.

4. Johansen KL, Shubert T, Doyle J, Soher B, Sakkas GK, Kent-Braun JA. Muscle atrophy in patients receiving hemodialysis: Effects on muscle strength, muscle quality, and physical function. Kidney Int. 2003;63(1):291-7.

5. Segura-Ortí E, Gordon PL, Doyle JW, Johansen KL. Correlates of Physical Functioning and Performance Across the Spectrum of Kidney Function. Clin Nurs Res. 2018;27(5):579-96.

6. Portilla Franco ME, Tornero Molina F, Gil Gregorio P. La fragilidad en el anciano con enfermedad renal crónica. Nefrologia. 2016;36(6):609-15.

7. Pérez Domínguez FB. Comparación de los efectos de un programa de ejercicio intradiálisis frente a un programa de ejercicio domiciliario. [Valencia]: Universidad Cardenal Herrera; 2016. Disponible en: https://www.educación.gob.es/teseo/mostrarRef. do? ref $=1695039$ 
8. Esteve Simo $\mathrm{V}$, Junqué Jiménez $A$, Moreno Guzmán F, Carneiro Oliveira J, Fulquet Nicolas M, Pou Potau $M$ et al. Beneficios del ejercicio físico de baja intensidad durante la sesión de hemodiálisis en el paciente anciano. Nefrologia. 2015;35(4):385-94.

9. Esteve Simó $V$, Junqué $A$, Fulquet $M$, Duarte $V$, Saurina A, Pou M et al. Complete Low-Intensity Endurance Training Programme in Haemodialysis Patients: Improving the Care of Renal Patients. Nephron Clin Pract. 2014;128(3-4):387-93.

10. Segura-Ortí E. Fisioterapia sobre ejercicio en pacientes en hemodiálisis. Fisioterapia. 2017;39(4):137-9.

11. Segura-Ortí E, Martínez-Olmos FJ. Test-retest reliability and minimal detectable change scores for sit-to-stand-to-sit tests, the six-minute walk test, the one-leg heel-rise test, and handgrip strength in people undergoing hemodialysis. Phys Ther. 2011;91(8):1244-52.

12. Ortega Pérez de Villar L, Antolí García $S$, Lidón Pérez MJ, Amer Cuenca JJ, Benavent Caballer V, Segura Ortí E. Comparación de un programa de ejercicio intradiálisis frente a ejercicio domiciliario sobre capacidad física funcional y nivel de actividad física. Enferm Nefrol. 2016;19(1):45-54.

13. Hurvitz EA, Richardson JK, Werner RA. Unipedal stance testing in the assessment of peripheral neuropathy. Arch Phys Med Rehabil. 2001;82(2):198-204.

14. Podsiadlo D, Richardson S. The timed «Up \& Go»: a test of basic functional mobility for frail elderly persons. J Am Geriatr Soc. 1991;39(2):142-8.

15. Acquistapace F, Piepoli MF. The Walking Test: Use in clinical practice. Monaldi Arch Chest Dis [Internet]. 2016 [Consultado 4 noviembre 2017];72(1). Disponible en: http://www.monaldi-archives.org/index.php/macd/article/view/336
16. Lucia Ortega. Comparation of two exercise programs for hemodialysis patients, intradialysis vs home based program. Absolute and relative reliability of physical performance. [Valencia]: Universidad Cardenal Herrera; 2017. Disponible en: https://www.educación.gob.es/teseo/mostrarRef. do? ref $=1693242$

17. Junqué Jiménez $A$, Esteve Simó $V$, Tomás Bernaveu E, Paz López Ó, Iza Pinedo G, Luceño Solé I et al. Neuromuscular electrostimulation: a new therapeutic option in the improvement of the physical condition of patients on hemodialysis. Enferm Nefrol. 2014;17(4):269-76.

18. Rubio Páez $S$, Esteve Simó $V$, Junqué Jiménez $A$, Tomás Bernabéu E, Paz López 0, Iza Pinedo G et al. Electroestimulación neuromuscular intradiálisis, fuerza muscular, capacidad funcional y composición corporal. Enferm Nefrol. 2015;18(4):273-81.

19. Pita Fernández S, Pértega Diaz S, Rodríguez Maseda E. La fiabilidadde las medicionesclínicas. Elanálisisdeconcordanciaàravariablesnuméricas.CadAten Primaria 2003;10:290-6

20. Martínez Curbelo G, Cortés C, E M, Fernández P, C A del. Metodología para el análisis de correlación y concordancia en equipos de mediciones similares. Rev Univ Soc. 2016;8(4):65-70.

21. Wilkinson TJ Phd, Xenophontos S Msc, Gould DW Phd, Vogt BP Phd, Viana JL Phd, Smith AC Phd et al. Test-retestreliability, validation, and "minimal detectable change" scores for frequently reported tests of objective physical function in patientswithnon-dialysis chronickidneydisease.PhysiotherTheory Pract.2018 Mar 30:1-12

Este artículo se distribuye bajo una Licencia Creative Commons Atribución-NoComercial 4.0 Internacional. https://creativecommons.org/licenses/by-nc/4.0/

Open Access (c) (7) (8) 